\title{
Tendencias en el mercado de teléfonos móviles en el segmento de estudiantes universitarios en México: una aplicación del modelo de Markov*
}

\author{
Karina Paola MENDOZA YAMASHIRO \\ Nielsen \\ kmendozay@gmail.com \\ Verónica Lorena HERNÁNDEZ HERRERA \\ Marketing|Mattel SLA \\ VeronicaLorena.Hernandez@Mattel.com \\ Rogerio DOMENGE \\ Departamento Académico de Administración \\ Instituto Tecnológico Autónomo de México. ITAM. México, D.F. \\ domenge@itam.mx
}

Recibido: 24-02-2012

Aceptado: 19-10-2012

\section{RESUMEN}

El sector de telefonía móvil en México ha tenido un gran crecimiento en los últimos años, determinado por una dinámica competitiva muy agresiva entre las empresas participantes, tanto las operadoras como las marcas manufactureras de teléfonos. En este artículo se presenta un análisis de las tendencias de participación de mercado de las empresas integrantes del segmento de estudiantes universitarios que poseen teléfonos móviles en la ciudad de México. Se toma como base una muestra de 518 sujetos pertenecientes a nueve universidades. Se presenta un análisis de escenarios tendenciales basado en la aplicación del modelo de cadenas de Markov en el segmento total y en diversos sub segmentos. Los resultados del análisis sugieren que las operadoras y las marcas se comportan de manera distinta, tanto en su estructura competitiva, como en la velocidad de cambio de los clientes y el horizonte de tiempo que tardan en llegar a la participación en equilibrio. El dinamismo migratorio entre empresas es mayor entre marcas que entre operadoras. Asimismo, se presentan algunas pautas que pueden permitir diseñar estrategias que permitan modificar el comportamiento de cambio de las participaciones de las empresas en esta industria.

Palabras clave: Telefonía móvil, Análisis de escenarios, Modelo de cadenas de Markov, Lealtad de marca, Segmento de estudiantes universitarios, México.

\footnotetext{
${ }^{*}$ Se agradece a la Asociación Mexicana de Cultura, A.C. por el apoyo en la elaboración de la presente investigación.
} 


\title{
Mobile Phones Market Trends in the Mexican Undergraduate Students Segment: A Markov Chain Model Application
}

\begin{abstract}
Mexican mobile phone industry has been rapidly changing and growing in the last years due to the dynamic competition among mobile phone operators and mobile phone manufacturers. In this article, it is presented a market share tendency analysis of the main mobile phone operators and manufacturers inside Mexico City undergraduate students market. For the analysis it was taken a sample size of 518 individuals from nine different universities. The market share tendency analysis is based on the application of the Markov Chains model in the complete segment and in different sub segments. The results of this analysis suggest that operators and mobile phone manufacturers behave differently from each other; in competitive structure as well as in speed of customer change inside each market. It is shown that changes between mobile phone brands are more dramatic than changes between mobile phone operators. As well, both industries have different time periods to reach equilibrium between market shares. In this article, also different statements are exposed in order to allow the design of marketing strategies that can modify market shares tendencies for both industries.
\end{abstract}

Keywords: Mobile phones, Scenario analisis, Markov chain model, Brand loyalty, Undergraduate students segment, Mexico.

Sumario: 1. Introducción. 2. El sector de telefonía móvil en México. 3. El modelo. 4. Aplicación y resultados. 5. Discusión y conclusiones. Bibliografía.

\section{INTRODUCCIÓN}

El sector de telefonía móvil en México y en particular el segmento de estudiantes a nivel universitario con teléfono móvil, ha aumentado significativamente en la última década, únicamente del año 2010 al 2011, su crecimiento fue de $10 \%$ (CIU, 2011). En la ciudad de México la penetración de móviles alcanzó el 96\% de la industria telefónica en el año 2011, con un total de poco más de 95 millones de líneas móviles. Se espera que en 2014 se tenga una penetración del 100\% (CIU, 2011).

Los teléfonos móviles han evolucionado de ser productos de lujo a bienes de consumo masivo, a precios cada vez más accesibles. Actualmente los teléfonos móviles se han convertido en algo más que solo dispositivos de comunicación, ahora son usados cada vez más para actividades con fines personales, de trabajo y entretenimiento, haciendo que su demanda aumente año con año, sobre todo en segmentos más jóvenes. En el 2009, los tres países que alcanzaron la mayor suscripción de usuarios fueron Rusia, Italia y Finlandia; por cada 100 habitantes, cada país mencionado respectivamente alcanzó penetraciones de mercado de $163.6 \%, 151.4 \%$ y $144.6 \%$ (BMI, 2010).

Existe un sector del mercado que demanda dispositivos cada vez más sofisticados que los ayuden a simplificar sus tareas diarias. Del total del mercado 
de dispositivos adquiridos, $20 \%$ son los llamados "teléfonos inteligentes" o "smartphones", que se definen como celulares que ofrecen más características que un celular común, ya que permite organizar información, navegar en Internet, descargar aplicaciones e ingresar a redes sociales. Éstos alcanzaron un crecimiento del $96 \%$ a nivel global en el último trimestre del 2010 comparado con el año anterior. Las marcas que lideran este mercado son Android de Google, seguida por Apple y su dispositivo iPhone y RIM (Research in Motion) con Blackberry (Gartner, 2010).

El líder del mercado a nivel global por segundo año consecutivo en el mercado de la telefonía celular es Nokia con más de 117 millones de unidades vendidas alrededor del mundo (28\% de participación de mercado), seguida por Samsung con $17.2 \%$ de participación y LG con $6.6 \%$ (Gartner, 2010). Como resultado, el mercado que se presenta actualmente es un mercado cada vez más polarizado, ya que por un lado existe una demanda por dispositivos a precios muy accesibles y por otro, una demanda por dispositivos sofisticados y con tecnología de punta que incluya las últimas aplicaciones del mercado.

En México el mercado de servicios de telefonía móvil ya no crece tan rápido como lo hacía hace 5 años, cada vez hay menos suscriptores nuevos. Sin embargo, la penetración nacional aún se considera baja comparada con países latinoamericanos como Brasil y Chile, lo que significa que aún existen oportunidades de crecimiento.

La distribución de participación de mercado está concentrada solo en unas cuantas operadoras dando como resultado, según un estudio de la Organización para la Cooperación y Desarrollo Económicos (OCDE), que México en el 2009 haya sido el sexto país a nivel mundial con las tarifas más caras en la provisión de servicios de telefonía móvil (Euromonitor International, 2010a).

Al cierre del tercer trimestre del 2010 , el $86.6 \%$ de los usuarios de teléfonos móviles habían adquirido su celular bajo pre pago (compran tarjetas pre pagadas o realizan recargas), probablemente como una forma de limitar el gasto. El resto, $13.4 \%$, lo habían hecho bajo un contrato de post pago (pagan por medio de contratos post pago), modalidad que ha ido en aumento poco a poco desde el 2008 (COFETEL, 2010). Esta distribución de pre y post pago también se puede deber al bajo poder adquisitivo que tienen la mayoría de los mexicanos pertenecientes a niveles socioeconómicos $\mathrm{C}$ y $\mathrm{D}+$. Estos sectores juntos representan los segmentos más grandes y representativos de la sociedad mexicana, el nivel socioeconómico $\mathrm{C}$ representa aproximadamente el $17 \%$ y el D+ $36.4 \%$ de la población (Niveles Socioeconómicos AMAI, 2009). Esto conlleva a que el mercado mexicano tarde más que otros en valorar equipos más recientes y con mayor desarrollo tecnológico. De acuerdo con Euromonitor International (2010a), una de las variables más importantes que toman en cuenta los consumidores mexicanos a la hora de elegir un teléfono móvil es el precio. Según un estudio realizado en el 2006 a un total de 1200 mexicanos, a través de la agencia de investigación Parametría, a 
medida que aumenta el ingreso de las personas aumenta la posesión de celulares (Abundis, 2006).

Por otro lado, hay un porcentaje creciente del mercado de niveles socioeconómicos $\mathrm{A} / \mathrm{B}$ y $\mathrm{C}+$ que representan el $21 \%$ de la población mexicana (7.6\% y $13.7 \%$ respectivamente) que están adoptando una nueva tendencia en telefonía celular: los "teléfonos inteligentes" o "smartphones" y se espera que la participación de mercado de estos crezca paulatinamente empujada por este sector. Esta tendencia de adopción también se relaciona con la edad de los usuarios, ya que la tenencia de teléfonos móviles es mayor entre los jóvenes y mantiene una relación inversa con la edad, es decir que a mayor edad, menor posesión (Abundis, 2006).

Hoy en día, México cuenta con la infraestructura necesaria y regulaciones para permitir que se tenga acceso a la tecnología más avanzada de la telefonía móvil como lo es el servicio 3G (tercera generación), el cual ofrece un sistema de transmisión de datos de mayor rapidez y con servicios multimedia de alta calidad. Incluso, se discute la posibilidad de prepararse para una mayor expansión de los servicios 4G (cuarta generación) (BMI, 2011).

Por otro lago, el sector universitario en la ciudad de México representa un mercado en crecimiento de teléfonos móviles, lo cual resulta de interés tanto para las empresas operadoras como para las marcas manufactureras. Conocer la dinámica y tendencias de las participaciones de mercado de las empresas participantes en el segmento universitario es una etapa básica en el proceso de planeación de dichas empresas, lo cual permite identificar y analizar escenarios de referencia que conduzcan a explorar y contrastar diferentes escenarios alternos en función de diferentes estrategias para cada empresa y sus competidores. A partir de lo anterior, se plantea como objetivo de la presente investigación, determinar cuáles son los escenarios tendenciales de participación de las diferentes empresas operadoras y marcas manufactureras, participantes en la industria de telefonía móvil en el sector de jóvenes universitarios en la ciudad de México.

La estructura del artículo es la siguiente: en la sección dos se presentan los principales datos del sector de la telefonía móvil en México, incluyendo a los proveedores de servicios y a las principales marcas de teléfonos móviles en México. En la sección tres se plantea el modelo probabilístico de Markov, base teórica del análisis a realizar posteriormente. En la sección cuatro se presentan las características del instrumento utilizado para la recopilación de datos, la descripción de la población, el método de muestro utilizado y los resultados obtenidos de la aplicación del modelo. Por último, se presentan algunas implicaciones para la toma de decisiones y las conclusiones de la investigación. 


\section{EL SECTOR DE LA TELEFONÍA MÓVIL EN MÉXICO}

\section{Proveedores de servicios de telefonía móvil en México}

Las cuatro compañías operadoras más importantes de telefonía móvil en México son: Telcel, que bajo la operación de América Móvil, es el incuestionable líder del mercado de telefonía móvil en México, con una participación nacional del 71\%; seguido por Telefónica Móviles México (Movistar) que ha aumentado su participación en los últimos años con estrategias enfocadas a contrarrestar la demanda del líder y hacerla suya, cuenta con el $21.1 \%$. Iusacell, una empresa decidida a regresar al mercado con mucha más agresividad tiene el $4.2 \%$ y Nextel cuenta con el 3.7\% (BMI, 2011).

Telcel: Subsidiaria en México de la compañía de telecomunicaciones más grande de Latinoamérica perteneciente al Grupo Carso y propiedad del magnate mexicano Carlos Slim, América Móvil. Telcel fue fundada en 1989 y actualmente es, por mucho, la operadora líder en el mercado de telefonía móvil en México; cuenta con una penetración de mercado de casi el 80\% (BMI, 2011). Al final del primer semestre del 2010, Telcel proporcionaba servicio a más de 61 millones de suscriptores y en agosto de 2011 a casi 67 millones (AM, 2012), siendo el 90.7\% de estos usuarios de pre pago y el resto de post pago. A pesar que su participación de mercado ha comenzado a mostrar una tendencia decreciente, aún mantiene una ventaja de más de 50 puntos porcentuales sobre Movistar, su competidor más cercano (BMI, 2011).

Telcel, al ser la compañía dominante en el mercado de telefonía móvil en México constituye junto con Telmex, la empresa líder en el servicio de telefonía fija y principal proveedor de servicios de internet también en México, una casi fórmula monopólica que ha conducido a que los precios en la industria de las telecomunicaciones sean altos comparados con otros países de la OCDE (Euromonitor International, 2010a). A pesar de ser un mercado altamente concentrado, no existe una discriminación notoria entre los fabricantes de teléfonos móviles para distribuir sus equipos entre las operadoras en México, ya que existen varios acuerdos entre ellas y las marcas para ofrecer alternativas diferentes a los suscriptores. Esta empresa también domina el mercado de los centros de distribución y además ha conseguido beneficiarse de las ventas de celulares en la cadena de tiendas Sanborns, pertenecientes al Grupo Carso. Tiene convenios con las marcas más conocidas de equipos móviles en México y en los últimos años debido al avance tecnológico en los celulares, decidió establecer alianzas estratégicas con marcas reconocidas como Apple, RIM, Microsoft y Palm para distribuir exclusivamente el iPhone, modelos de Blackberry, el Windows Mobile y el Treo respectivamente. Aunque recientemente, sus competidores han comenzado a distribuir algunos modelos también, esto aún no parece ser una amenaza para 
Telcel, ya que cuenta con una variedad de diez marcas para ofrecer con diferentes modelos cada una, mientras que Movistar y Iusacell tienen cuatro o cinco únicamente (Euromonitor International, 2010b).

En febrero del 2011, comenzó para Grupo Carso una confrontación con las dos televisoras más importantes del país, Televisa y TV Azteca al disminuir la cantidad de publicidad contratada para este año, dando como resultado que los spots de las empresas que conforman a este Grupo (Telmex, Telcel, Sanborns y Sears, entre otras) no se anunciaran en televisión abierta por un año entero. Esto ha comenzado a generar diversas especulaciones sobre prácticas monopólicas de Telcel y Telmex, distintas quejas por competidores como Iusacell o Movistar a la COFETEL y una clara desventaja para Telcel y Telmex con respecto a sus competidores, ya que ellos tienen acceso a los espacios publicitarios en TV abierta que antes tenía este consorcio (CNN Expansión, 2011).

Telefónica: Establecida en 1998, Telefónica (Movistar) es la segunda operadora móvil más grande del país, después de Telcel. Desde abril del 2005, es parte de la compañía multinacional originaria de España, Telefónica Movistar. También en esa fecha se fijó un nuevo objetivo, alcanzar la tercera parte del mercado mexicano para el 2011 al aprovechar la portabilidad numérica vigente en el país desde el 2008 para obtener más fácilmente parte del portafolio de clientes de Telcel. Sin embargo, su participación de mercado ha crecido a paso lento, alcanzando subir $1 \%$ cada año desde el 2008. Su estrategia está basada en ofrecer mayor variedad de servicios que su competidor más fuerte, pero esto apenas ha sido percibido por los usuarios. A finales de junio del 2010, reportó más de 18 millones de suscriptores a nivel nacional, de los cuales el $92.6 \%$ son consumidores de servicio de pre pago y $7.4 \%$ de post pago (BMI, 2011).

Nextel: Esta compañía de telefonía móvil caracterizada por ofrecer el servicio PTT (Push To Talk), comenzó sus operaciones en México en 1998 después de adquirir Corporación Mobilcom en 1996, la cual ofrecía en el país el servicio de radiocomunicación móvil especializado en grupos o flotillas desde 1994. En el año 2000 es cuando Nextel comienza su verdadera expansión a nivel nacional gracias al surgimiento del crossfleet, una tecnología en radiocomunicación que permite comunicarse entre usuarios de diferentes flotillas o grupos (Nextel México, 2011). Nextel cuenta con cobertura en todos los estados de la República Mexicana, aunque no en todas las ciudades de estos y ha enfocado su estrategia en el sector empresarial. La compañía Motorola es proveedor de su red de radiocomunicación (Nextel México, 2011). A finales del primer semestre del 2010, Nextel contaba con más de tres millones de usuarios, convirtiéndose en el competidor más cercano para Iusacell. Una de las principales ventajas que ofrece es que todas las llamadas que sus usuarios realizan se cobran como llamadas locales, sin importar que sea a larga distancia o a móviles, esto es una característica que ninguna otra compañía de telefonía móvil ofrece aún. También ha comenzado a promocionar su Plan Conexión PC, enfocado a quienes desean seguir comunicados con su red de 
contactos por medio de su computadora cuando se encuentren fuera de cobertura (Nextel México, 2011). El 100\% de sus suscriptores tienen un servicio de post pago, sin embargo la compañía se ha dado cuenta del potencial que podría tener el servicio de radiocomunicaciones en los sectores medio y medio-bajo del país por lo que ha comenzado a realizar pruebas piloto para conocer las oportunidades que tendría al ofrecer servicios de pre pago a estos segmentos (BMI, 2011).

Iusacell: Comenzó sus operaciones en 1992, aunque fue establecida desde 1989 por Grupo IUSA. A principios de los años noventa llegó a ocupar la posición de líder del mercado, sin embargo después de la crisis económica del 1995, perdió clientes al ofrecer únicamente el servicio de post pago y se convirtió en la segunda operadora del país. Desde entonces ha ido perdiendo terreno en el mercado y con la entrada de Movistar, fue rezagada a la tercera posición. Actualmente es manejada por Grupo Salinas, en el 2007, adquirió la compañía de telefonía móvil Unefon, también operada por el grupo del magnate mexicano Ricardo Salinas Pliego, con el objetivo de obtener mayor cobertura a nivel nacional y convertirse juntas en el tercer operador móvil en México.

En el 2009, se tomó la decisión de que cada marca debía funcionar de manera independiente para enfocarse en sus mercados objetivos respectivos: Iusacell a niveles socioeconómicos (NSE) A/B y C+, especialmente a ejecutivos, y Unefon a los segmentos de NSE C, D+y D/E. Estas dos marcas cuentan con un aproximado de 3.5 millones de usuarios en el país, siendo el 68\% suscriptores de servicio de pre pago y $32 \%$ de post pago (BMI, 2011). En la actualidad también opera la conexión a Internet inalámbrica y servicios de computadora en siete de las nueve regiones de México, incluyendo la Ciudad de México, Monterrey, Guadalajara, Tijuana, Acapulco, Puebla, León, Mérida, entre otras. En el 2008, Iusacell era la única de las compañías que ofrecía tecnología 3G para todos sus usuarios en el país y en ofrecer tecnología CDMA (Code Division Multiple Access) en México, lo cual genera máxima eficiencia en el espectro, es decir, cualquier tipo de interferencia es evitada. Sin embargo, aún no consigue la cobertura que ofrecen sus competidores. Además, en el 2009, Telcel también logró ofrecer a sus usuarios tecnología 3G (Euromonitor International, 2010b).

Un nuevo panorama se avecina para la industria de telecomunicaciones que estará cambiando por completo el terreno de la competencia en el sector de la telefonía móvil. En abril del 2011, Grupo Televisa en común acuerdo con Grupo Salinas, decide aliarse con Iusacell para darle fuerza a esta operadora y al mismo tiempo entrar al mercado de la telefonía móvil con un 50\% de participación de las acciones (1,600 millones de dólares) (Excélsior, 2011). Por otro lado, en agosto del 2011, Telcel (América Móvil) anunció la oferta de compra del 40\% de Teléfonos de México con el objetivo de poder brindar el llamado "cuádruple play" o "total play": telefonía móvil, fija, internet y televisión. Por lo anterior, se podría decir que Nextel, Unefon y Movistar no podrían representar una competencia real para 
Televisa-Iusacell y Telcel-Telmex al no operar redes de telefonía fija y móvil que ofrezcan voz, video y datos para converger en un total play (Reforma 2011).

\section{Principales marcas de teléfonos móviles en México}

La participación de mercado de las distintas marcas de teléfonos móviles en México, en el año 2010, se encontraba liderada por Nokia con un $28 \%$ del mercado, seguida por Motorola con un 26\%, LG con un 25\%, Sony Ericsson con un $7.3 \%$, Samsung con un $3.6 \%$ y Apple con $1.6 \%$, el resto de las marcas no representaban una participación importante aún (Euromonitor International, 2010a).

Nokia: Líder del mercado en México y una de las empresas de tecnología de telecomunicaciones más grandes en el mundo. Latinoamérica representa el 10\% de sus ventas. A pesar de que sus teléfonos móviles de bajo costo han tenido éxito en la región, la popular introducción de los "smartphones" y computadoras cada vez más innovadoras han beneficiado a sus competidores como RIM y Google con Android. El sistema operativo de Nokia, Symbian, ha perdido su liderazgo en el mercado y caído al tercer lugar (BMI, 2011). Una de las razones del éxito de Nokia se debe a su lanzamiento de una gran variedad de equipos dirigidos a varios segmentos (Euromonitor International, 2011a).

Motorola: Es una empresa estadounidense que se ha mantenido como un fuerte competidor en Latinoamérica. Sin embargo, en México y a nivel mundial su participación ha ido cayendo los últimos años. Últimamente se ha enfocado a promover los teléfonos con servicio $3 \mathrm{G}$, viendo gran potencial en el mercado latinoamericano, pero no consiguió prever la evolución de la tecnología en smartphones por parte de competidores que han ido aumentando su participación, como Apple, LG y RIM (Euromonitor International, 2011). A mediados de Agosto del 2011, Google Inc. cerró un trato por \$12.5 millones de dólares por la compra de Motorola. Actualmente Google Inc. además de ser el motor de búsqueda de información más grande y más utilizado a nivel mundial, es proveedor del software: Android, sistema operativo de smartphones de marcas como LG Electronics y Samsung. Con la compra de Motorola, Google incursionará en ser proveedor también de hardware de telefonía móvil, con lo cual podrá ser un competidor más cercano de Apple Inc. y Blackberry hecho que puede cambiar el terreno de juego de la industria de telefonía móvil a nivel mundial (Wall Street Journal Americas, 2011).

$L G$ Electronics: Empresa Coreana (Sur) y tercera más grande en participación de mercado. Fabrica gran variedad de productos electrónicos, teléfonos móviles y productos petroquímicos. En México ocupa el 25\% del mercado, disputando el segundo sitio con Motorola (Euromonitor International, 2011). Así como sus principales competidores, tiene convenio con las grandes operadoras de telefonía móvil en México: Telcel, Iusacell y Movistar. Ha invertido muchos recursos en los 
últimos años para lograr posicionarse en el mercado mexicano, En el 2007 invirtió cerca de 50 millones de pesos mexicanos con el objetivo de alcanzar 3 millones de equipos vendidos. Actualmente produce equipos con la tecnología más avanzada para competir con equipos como el iPhone de Apple (Euromonitor International, 2011).

Samsung Electronics: También es una empresa líder en fabricación de productos electrónicos y de telefonía móvil originaria de Corea del Sur. Es una empresa cuya principal estrategia en los últimos años ha sido imitar algunos diseños o tecnología de sus competidores y ofrecerla en una forma más práctica de usar. Los equipos se ofrecen a un precio más bajo, considerados de buena calidad. También ofrece smartphones, los cuales utilizan el sistema operativo de Google, Android, considerado por los consumidores a nivel global el mejor sistema operativo para smartphones. Esta compañía ha declarado que desea ser un fuerte competidor para Apple, especialmente ahora que dicha empresa atraviesa por cambios en su dirección corporativa (CNN Expansión, 2011).

Sony Ericsson Mobile Communications AB: Es un joint venture que se formó en el 2001, entre la empresa sueca Ericsson y la firma japonesa Sony. Ofrece gran variedad de equipos desde su introducción al mercado en el 2002 y actualmente cuenta con servicios 3G y smartphones con sistema operativo Android. En México su participación de mercado es relativamente baja, con un $7.3 \%$ del mercado en el 2009 y con expectativas a disminuir (Euromonitor International, 2011). Sin embargo, sus equipos siempre han resultado innovadores y con algunos diferenciadores que los hacen únicos en el mercado.

Apple: Empresa de origen estadounidense enfocada al desarrollo de tecnología para los productos electrónicos que fabrica y posicionada como una de las marcas más reconocidas a nivel mundial, gracias a su constante innovación en el mercado de los reproductores de MP3 (iPod), computadores personales (iBook), telefonía móvil (iPhone) y los "tablet" PC (iPad). Su valor de mercado es de 320,000 millones de dólares (Excélsior, 2011). Dentro de la telefonía móvil, la introducción del iPhone en el 2007, revolucionó la manera en que las compañías fabricantes llevaban a cabo sus avances en diseño y tecnología móvil. A pesar de no ser un teléfono de bajo costo, su popularidad alrededor del mundo ha ido en aumento, al pasar de una participación de mercado en el 2009 del 2.5\% al 3.2\% en el tercer trimestre del 2010 (Gartner, 2010). En el primer trimestre de 2012, Apple contaba con el 50\% de smartphones en Estados Unidos (AppleWeblog, 2012). En México, la primera generación del iPhone fue introducida en el 2008 únicamente por Telcel, asimismo la segunda, tercera y cuarta generación. Sin embargo, ya existe un convenio con Iusacell desde el 2010 para que sea otro distribuidor de este teléfono móvil. Su participación en el mercado mexicano durante el 2009 fue de $1.5 \%$ (Euromonitor International, 2010a).

RIM (Research in Motion): Empresa de origen canadiense famosa por la creación del sistema operativo Blackberry y por la fabricación de los teléfonos 
móviles inteligentes con el mismo nombre. Su participación a nivel mundial en tercer trimestre del 2010 fue de $2.9 \%$, casi sin variación respecto al mismo trimestre en el 2009 (Gartner, 2010). En México, cuenta con convenios con todas las operadoras en México, siendo Telcel y Nextel sus socios más fuertes. Se ha convertido en el favorito de jóvenes empresarios por la facilidad de administrar su agenda (Euromonitor International, 2010b).

\section{EL MODELO}

El modelo de cadenas Markov de primer orden es un sistema lineal de ecuaciones que representa una serie de estados discretos del sistema a lo largo del tiempo. Cada cambio, transición o iteración del sistema está en función del estado anterior, sin memoria, asumiendo una probabilidad de cambio constante a lo largo del tiempo. La probabilidad de que ocurra un evento depende del evento inmediato anterior (Levin y Kirkpatrick, 1978). El modelo de cadenas de Markov es una representación del comportamiento de una cadena de eventos mediante un modelo estocástico (item Software, 2012).

El modelo (Lilien \& Rangaswamy, 2006) de cadenas de Markov (Ching, et al., 2003; Lipstein, 1965, Styan y Smith, 1964; Ehrenberg, 1965) ha tenido varias aplicaciones en mercadotecnia como en el modelaje del comportamiento del consumidor y la participación de mercado (Vilcassim y Dipak, 1991; Givon y Horsky, 1978; Herniter y Magee, 1961), la segmentación de mercado (Blattberg y Sen, 1976), la selección y lealtad de marca (Ching, et al., 2004; Whitaker, 1978; Herniter, 1971; Harary y Lipstein, 1962) y la migración (Kelley y Weiss, 1969) entre otras.

En este estudio, se modelan los cambios que tienen las participaciones de mercado de las compañías operadoras de telefonía móvil y las de marcas manufactureras de teléfonos móviles, en función de sus probabilidades de cambio obtenidas en forma empírica.

El modelo de Markov se define como:

\section{Donde:}

$$
P_{t+1}=P_{t} T_{i j}
$$

$\mathrm{P}_{\mathrm{t}}$ : vector de participaciones de mercado en el periodo $\mathrm{t}$ (discreto) para $\mathrm{t}=$ $1 \ldots \mathrm{m}$

$\mathrm{T}_{\mathrm{ij}}$ :matriz de probabilidades de transición de la empresa i a la empresa j del periodo $\mathrm{t}$ al período $\mathrm{t}+1$, con dimensión $\mathrm{m} \mathrm{x} \mathrm{m}$ m:número de empresas participantes en el mercado 
El modelo supone que la matriz de transición es constante para todo período considerado y sigue un proceso Markov de primer orden, es decir, que la participación del periodo t está en función solamente del periodo anterior t-1 (Levin y Kirkpatrick, 1978). La Figura 1 presenta la estructura del modelo para tres marcas, la cual puede generalizarse para m marcas o empresas participantes en un determinado mercado.

A partir del modelo planteado, es posible obtener un vector de equilibrio de las participaciones de mercado, el cual indica el estado final de largo plazo de dichas participaciones. Este vector se obtiene resolviendo el sistema de ecuaciones simultáneas basadas en la matriz de transición incluyendo la restricción de la suma de participaciones igual al total del mercado y considerando que el vector de participaciones conserva un mismo valor de una iteración a la siguiente.

$$
\begin{aligned}
& P E=P E T_{i j} \\
& \Sigma^{\mathrm{m}} \mathrm{PE}_{\mathrm{t}}=1
\end{aligned}
$$

Donde:

PE: vector de participaciones en equilibrio de mercado de $m$ participantes $\mathrm{T}_{\mathrm{ij}}$ :matriz de probabilidades de transición de la empresa i a la empresa $\mathrm{j}$ del periodo $\mathrm{t}$ al período $\mathrm{t}+1$, con dimensión $\mathrm{m} \mathrm{x} \mathrm{m}$ m:número de empresas participantes en el mercado

Figura 1. Diagrama de transición de estado o estructura del modelo Markov

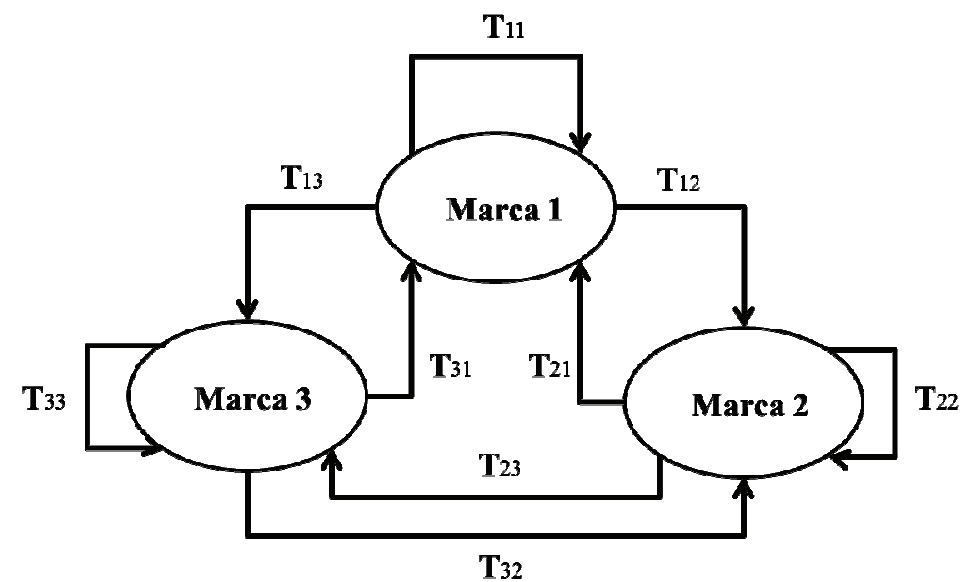


El sistema de ecuaciones se resuelve para PE de la siguiente forma:

$$
\begin{gathered}
P E=P E T_{i j} \\
P E\left[T_{i j}-I\right]=0 \\
P E=\left[T_{i j}-I\right]^{-1}
\end{gathered}
$$

Donde:

I:Matriz identidad, con dimensión m x m

Debido a que es necesario tener $\mathrm{m}$ ecuaciones independientes para resolver el sistema lineal, resulta necesario substituir una de ellas con la restricción de participación de mercado de exhaustividad (Herniter \& Magee, 1960):

$$
\Sigma^{\mathrm{m}} \mathrm{PE}_{\mathrm{t}}=1
$$

\section{APLICACIÓN Y RESULTADOS}

\section{Instrumento, población y muestra}

Se diseñó un cuestionario con el objetivo de recopilar información acerca del segmento de mercado de jóvenes universitarios en la ciudad de México, definido como jóvenes de entre 17 y 27 años, que poseen teléfono móvil. Se realizó un perfil general del segmento y una segmentación. Asimismo, se buscó obtener información sobre la participación de mercado y de sus tendencias de las empresas operadoras y manufactureras de teléfonos móviles participantes en el segmento.

El cuestionario aplicado se estructuró en varias secciones: a. identificación de las marcas de teléfonos móviles actual y anterior, causales y período de cambio; b. identificación de las empresas operadoras de telefonía móvil actual y anterior, causales y período de cambio; c. hábitos de compra; d. variables de segmentación.

La matrícula de estudiantes a nivel universitario en México, en el ciclo escolar 2010-2011, estaba por encima de los 3 millones. Las instituciones públicas cubrían el $67 \%$ de esta matrícula mientras las privadas el resto. Sin embargo, pese al número de estudiantes en instituciones públicas, de los 4,462 planteles universitarios que tuvo registrados la Secretaría de Educación Pública, 1,968 eran públicos y 2,494 privados (Tuirán, 2010). Se calcula que en la ciudad de México existen aproximadamente 1.4 millones de estudiantes de nivel universitario, representando casi el 16\% de la población de la capital del país (INEGI 2010). El segmento de jóvenes en edad universitaria representa el 3.3\% del total de usuarios 
de teléfonos móviles a nivel nacional y el $1.44 \%$ del mercado en la ciudad de México (INEGI 2010).

Se aplicaron 541 cuestionarios en distintas universidades de la ciudad de México, siguiendo un esquema aleatorio sistemático, de los cuales 267 fueron en universidades públicas y 274 en privadas (Tabla 1). Del total de participantes en la muestra, el $96 \%$ de contaba con un teléfono móvil y el $17.5 \%$ con dos o más y el $4 \%$ no contaba con móvil.

Tabla 1. Universidades que conforman la muestra inicial

\begin{tabular}{|l|c|c|}
\hline \multicolumn{1}{|c|}{ Universidad } & Tipo & $\begin{array}{c}\text { Número de } \\
\text { participantes }\end{array}$ \\
\hline EBC: Escuela Bancaria y Comercial & Privada & 37 \\
\hline IPN: Instituto Politécnico Nacional & Pública & 77 \\
\hline ITAM: Instituto Tecnológico Autónomo de México & Privada & 102 \\
\hline ITESM: Instituto Tecnológico de Estudios Superiores de Monterrey & Privada & 31 \\
\hline UAM: Universidad Autónoma Metropolitana & Pública & 93 \\
\hline UAS: Universidad Anáhuac del Sur & Privada & 45 \\
\hline ULSA: Universidad La Salle & Privada & 44 \\
\hline UNAM: Universidad Nacional Autónoma de México & Pública & 97 \\
\hline UP: Universidad Panamericana & Privada & 15 \\
\hline
\end{tabular}

Los cuestionarios se aplicaron a alumnos de las siguientes disciplinas: $42 \%$ al área Económico Administrativa, 35\% al área de Físico Matemáticas e Ingenierías, $12 \%$ a la rama de Humanidades y Ciencias Sociales y $12 \%$ al área de Ciencias Bilógicas y de la Salud. La edad media fue de 22.3 años.

\section{Aplicación del modelo de Markov}

En la Tabla 2 se muestra la matriz n (número de sujetos) correspondiente a las empresas operadoras, identificada a partir de la muestra, después de depurar la base de datos, obteniendo una muestra con datos completos de 518 sujetos. La matriz de transición $T_{\mathrm{ij}}$ de las empresas operadoras se presenta en la Tabla 3 y su vector de participación inicial $\mathrm{P}_{0}$ para $\mathrm{t}=0$ se encuentra a la derecha de dicha matriz. El vector $\mathrm{P}_{1}$ de participaciones en el período $\mathrm{t}=1$, se encuentra en el último renglón de la misma.

Telcel, líder del segmento, incrementó seis puntos porcentuales su participación de mercado mientras que Movistar perdió 5 puntos. En la primera iteración Iusacell logró escalar, dejando atrás a Nextel. A partir de los datos de la encuesta se identifica que el $37 \%$ de los sujetos habían cambiado de operadora debido a que buscaban una mayor cobertura, el 33\% lo hacía porque la compañía a la que cambiaban ofrecía tarifas más bajas y el resto debido a que ofrecían mejor servicio al cliente y tenían mayor variedad de equipos. 
Tabla 2. Matriz n (número de sujetos) que cambian de empresas operadoras

\begin{tabular}{|c|c|c|c|c|c|c|}
\hline$n \mathbf{i j}$ & Telcel & Nextel & Iusacel & Movistar & Unefon & \\
\hline Telcel & 354 & 14 & 19 & 15 & 3 & 405 \\
\hline Nextel & 11 & 5 & 4 & 2 & 0 & 22 \\
\hline Ius acel & 13 & 0 & 4 & 1 & 0 & 18 \\
\hline Movistar & 46 & 0 & 1 & 8 & 1 & 56 \\
\hline \multirow[t]{2}{*}{ Unefon } & 13 & 0 & 0 & 3 & 1 & 17 \\
\hline & 437 & 19 & 28 & 29 & 5 & 518 \\
\hline
\end{tabular}

Tabla 3. Matriz de transición $\mathrm{T}_{\mathrm{ij}}$ de empresas operadoras

\begin{tabular}{|c|c|c|c|c|c|c|}
\hline Tij & Telcel & Nextel & Iusacel & Movistar & Unefon & \\
\hline Telcel & $87.41 \%$ & $3.46 \%$ & $4.69 \%$ & $3.70 \%$ & $0.74 \%$ & $\mathbf{7 8 . 1 9 \%}$ \\
\hline Nextel & $50.00 \%$ & $22.73 \%$ & $18.18 \%$ & $9.09 \%$ & $0.00 \%$ & $\mathbf{4 . 2 5 \%}$ \\
\hline Iusacel & $72.22 \%$ & $0.00 \%$ & $22.22 \%$ & $5.56 \%$ & $0.00 \%$ & $\mathbf{3 . 4 7 \%}$ \\
\hline Movistar & $82.14 \%$ & $0.00 \%$ & $1.79 \%$ & $14.29 \%$ & $1.79 \%$ & $\mathbf{1 0 . 8 1 \%}$ \\
\hline Unefon & $76.47 \%$ & $0.00 \%$ & $0.00 \%$ & $17.65 \%$ & $5.88 \%$ & $\mathbf{3 . 2 8 \%}$ \\
\hline & $\mathbf{8 4 . 3 6 \%}$ & $\mathbf{3 . 6 7 \%}$ & $\mathbf{5 . 4 1 \%}$ & $\mathbf{5 . 6 0 \%}$ & $\mathbf{0 . 9 7 \%}$ & 1.000 \\
\hline
\end{tabular}

Asimismo, las matrices de transición n y $\mathrm{T}_{\mathrm{ij}}$, de las marcas, estimados a partir de la muestra, se presentan en las Tablas 4 y 5 . El vector de participación inicial $\mathrm{P}_{0}$ para $\mathrm{t}=0$ de mercado de las marcas se encuentra a la derecha de la matriz de la Tabla 5 y el vector $\mathrm{P}_{1}$ para $\mathrm{t}=1$ se encuentra en el último renglón de la misma tabla. 
Tabla 4. Matriz n (número de sujetos) que cambian de marca

\begin{tabular}{|c|c|c|c|c|c|c|c|c|c|}
\cline { 2 - 10 } \multicolumn{1}{c|}{} & Sony & & & iPhone & Blackberry & & \multicolumn{1}{c|}{} \\
\hline $\mathrm{n}$ & Ericsson & Nokia & Motorola & (Apple) & (RIM) & LG & Sams ung & Otro & \\
\hline Sony Ericss on & 18 & 41 & 11 & 7 & 39 & 21 & 9 & 9 & 155 \\
\hline Nokia & 30 & 30 & 11 & 6 & 47 & 8 & 7 & 3 & 142 \\
\hline Motorola & 13 & 14 & 5 & 5 & 28 & 5 & 5 & 2 & 77 \\
\hline iPhone (Apple) & 0 & 1 & 2 & 2 & 7 & 1 & 1 & 1 & 15 \\
\hline Blackberry (RIM) & 2 & 3 & 2 & 4 & 8 & 1 & 0 & 0 & 20 \\
\hline LG & 7 & 18 & 6 & 1 & 16 & 3 & 5 & 0 & 56 \\
\hline Sams ung & 2 & 10 & 3 & 1 & 5 & 2 & 2 & 2 & 27 \\
\hline Otro & 8 & 7 & 0 & 0 & 2 & 4 & 1 & 5 & 27 \\
\hline & 80 & 124 & 40 & 26 & 152 & 45 & 30 & 22 & 519 \\
\hline
\end{tabular}

Tabla 5. Matriz de transición $T_{i j}$ de marcas

\begin{tabular}{|c|c|c|c|c|c|c|c|c|c|}
\hline & Sony & & & iPhone & Blackberry & & & & \\
\hline $\mathrm{n}$ & Ericsson & Nokia & Motorola & (Apple) & (RIM) & LG & Sams ung & Otro & \\
\hline Sony Ericsson & $11.6 \%$ & $26.5 \%$ & \begin{tabular}{|l|}
$7.1 \%$ \\
\end{tabular} & $4.5 \%$ & $25.2 \%$ & $13.5 \%$ & $5.8 \%$ & $5.8 \%$ & $29.9 \%$ \\
\hline Nokia & $21.1 \%$ & $21.1 \%$ & $7.7 \%$ & $4.2 \%$ & $33.1 \%$ & $5.6 \%$ & $4.9 \%$ & $2.1 \%$ & $27.4 \%$ \\
\hline Motorola & $16.9 \%$ & $18.2 \%$ & $6.5 \%$ & $6.5 \%$ & $36.4 \%$ & $6.5 \%$ & $6.5 \%$ & $2.6 \%$ & $14.8 \%$ \\
\hline iPhone (Apple) & $0.0 \%$ & $6.7 \%$ & $13.3 \%$ & $13.3 \%$ & $46.7 \%$ & $6.7 \%$ & $6.7 \%$ & $6.7 \%$ & $2.9 \%$ \\
\hline Blackberry (RIM) & $10.0 \%$ & $15.0 \%$ & $10.0 \%$ & $20.0 \%$ & $40.0 \%$ & $5.0 \%$ & $0.0 \%$ & $0.0 \%$ & $3.9 \%$ \\
\hline LG & $12.5 \%$ & $32.1 \%$ & $10.7 \%$ & $1.8 \%$ & $28.6 \%$ & $5.4 \%$ & $8.9 \%$ & $0.0 \%$ & $10.8 \%$ \\
\hline Sams ung & $7.4 \%$ & $37.0 \%$ & $11.1 \%$ & $3.7 \%$ & $18.5 \%$ & $7.4 \%$ & $7.4 \%$ & $7.4 \%$ & $5.2 \%$ \\
\hline \multirow[t]{2}{*}{ Otro } & $29.6 \%$ & $25.9 \%$ & $0.0 \%$ & $0.0 \%$ & $7.4 \%$ & $14.8 \%$ & $3.7 \%$ & $18.5 \%$ & $5.2 \%$ \\
\hline & $15.4 \%$ & $23.9 \%$ & $7.7 \%$ & $5.0 \%$ & $29.3 \%$ & $8.7 \%$ & $5.8 \%$ & $4.2 \%$ & $100 \%$ \\
\hline
\end{tabular}

Inicialmente la marca líder del segmento en $\mathrm{t}=0$ era Sony Ericsson seguida de Nokia. En la primera iteración Sony disminuye considerablemente su participación en poco más de $50 \%$ y Blackberry se posiciona como líder incrementado su participación en ocho veces, seguida de Nokia.

Con el objetivo de identificar el período de cambio de cada iteración, para las empresas operadoras y para las marcas, se calculó el promedio del período de tiempo del último cambio en cada uno de estos rubros en el cuestionario. Los períodos calculados y considerados en el presente análisis son de 2.29 años para el período promedio de cambio de operadoras y de 1.25 años para el período promedio de cambio de marca de móviles.

En la diagonal mayor de las matrices de transición se puede identificar la marca y la operadora con mayor lealtad, es decir aquella con mayor valor $\mathrm{T}_{\mathrm{ij}}$. Las empresas con mayor lealtad son la operadora Telcel con $87.4 \%$ y la marca Blackberry con $40 \%$. LG y Motorola son las marcas con menor lealtad, así como Unefon y Movistar en el caso de las operadoras. 


\section{Participaciones en equilibrio}

Basadas en la matriz de transición y el vector de participación de mercado de las empresas operadoras y aplicando el modelo de Markov, en la Tabla 6 se presentan las participaciones de mercado de las siete primeras iteraciones, lo que equivale a aproximadamente 16 años de horizonte de análisis $(2.29 * 7)$.

Tabla 6. Participaciones de mercado de las empresas operadoras para las primeras siete iteraciones.

\begin{tabular}{|r|c|c|c|c|c|}
\cline { 2 - 6 } \multicolumn{1}{c|}{} & Telcel & Nextel & Ius acel & Movistar & Unefon \\
\hline Inicial & 0.7818533 & 0.0424710 & 0.0347490 & 0.1081081 & 0.0328185 \\
\hline $\mathbf{1}$ & 0.8436293 & 0.0366795 & 0.0540541 & 0.0559846 & 0.0096525 \\
\hline $\mathbf{2}$ & 0.8481420 & 0.0374988 & 0.0592584 & 0.0472842 & 0.0078166 \\
\hline $\mathbf{3}$ & 0.8477041 & 0.0378409 & 0.0606202 & 0.0462481 & 0.0075867 \\
\hline $\mathbf{4}$ & 0.8474490 & 0.0379036 & 0.0609460 & 0.0461500 & 0.0075514 \\
\hline $\mathbf{5}$ & 0.8473851 & 0.0379090 & 0.0610161 & 0.0461441 & 0.0075457 \\
\hline $\mathbf{6}$ & 0.8473734 & 0.0379080 & 0.0610295 & 0.0461443 & 0.0075448 \\
\hline $\mathbf{7}$ & 0.8473718 & 0.0379074 & 0.0610318 & 0.0461444 & 0.0075447 \\
\hline
\end{tabular}

De acuerdo a la solución del sistema de ecuaciones del modelo de Markov con la restricción de exhaustividad arriba planteada, la Tabla 7 presenta el vector de participación en equilibrio (PE) de las empresas operadoras de móviles.

Tabla 7. Vectores de participación en equilibrio de las empresas operadoras

\begin{tabular}{|c|c|}
\hline Telcel & $84.74 \%$ \\
\hline Nextel & $3.79 \%$ \\
\hline Iusacel & $6.10 \%$ \\
\hline Movistar & $4.61 \%$ \\
\hline Unefon & $0.75 \%$ \\
\hline
\end{tabular}

Las participaciones de mercado para las operadoras cambian significativamente en el equilibrio a partir de $\mathrm{t}=0$. Telcel incrementa su participación mayoritaria de $78.2 \%$ a $84.7 \%$ y Iusacel incrementa en más de $75 \%$ su participación, Movistar disminuye en más de $42 \%$ y Unefon en $23 \%$. La Figura 2 presenta gráficamente el comportamiento de las participaciones de las operadoras en el tiempo. 
Figura 2. Participaciones de mercado de las empresas operadoras
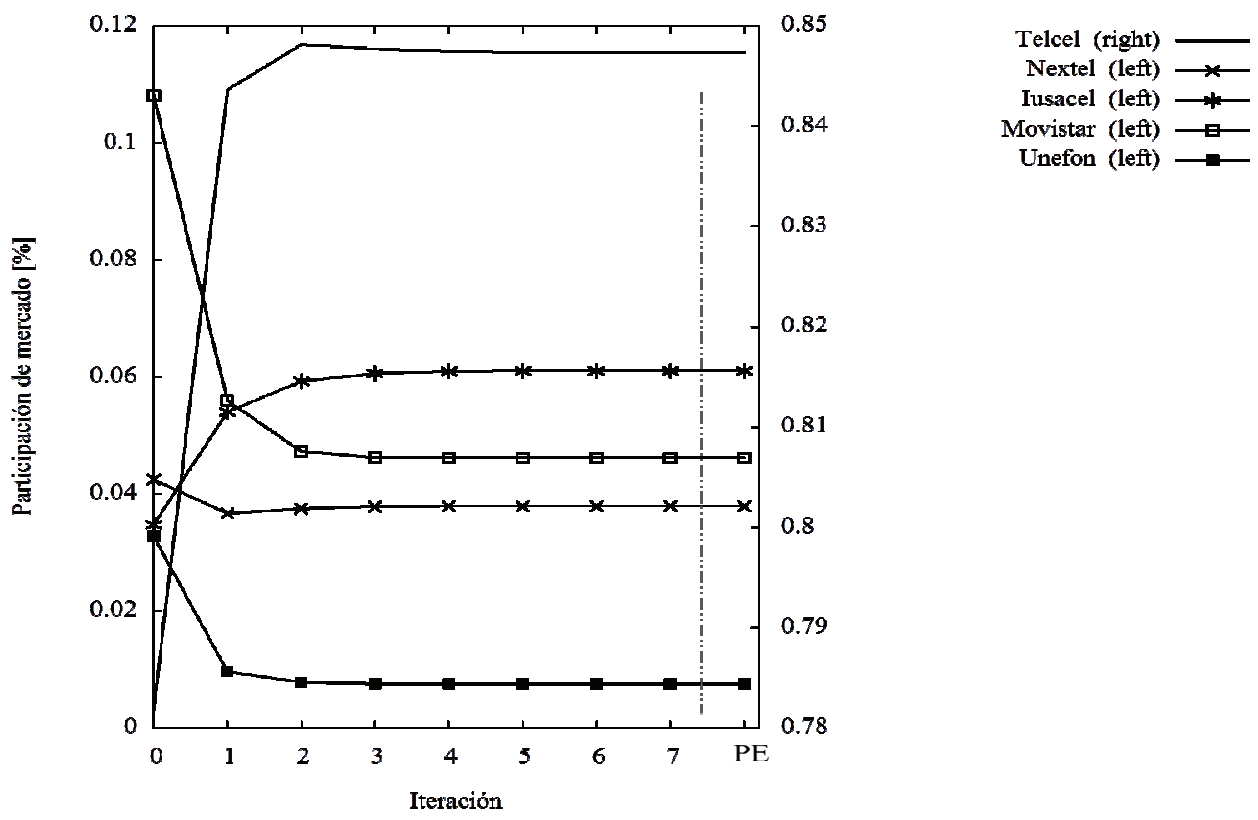

En la Tabla 8 se presentan las participaciones de mercado de las marcas de las siete primeras iteraciones, lo que equivale a un horizonte de casi nueve años (1.25 * 7). Las participaciones de mercado para las marcas cambian significativamente de los valores iniciales $(t=0)$ a los valores en equilibrio (Tabla 9). Blackberry logra convertirse en líder de este mercado aumentando más de nueve veces su participación. Sony Ericsson pierde su liderazgo. iPhone de Apple incrementa notablemente su participación en casi $400 \%$.

Tabla 8. Participaciones de mercado de las marcas para las primeras siete iteraciones.

\begin{tabular}{|c|c|c|c|c|c|c|c|c|}
\hline & \multirow{2}{*}{$\begin{array}{c}\text { Sony } \\
\text { Ericsson } \\
\end{array}$} & \multirow[b]{2}{*}{ Nokia } & \multirow[b]{2}{*}{ Motorola } & \multirow{2}{*}{$\begin{array}{c}\text { iPhone } \\
\text { Apple } \\
\end{array}$} & \multirow{2}{*}{\begin{tabular}{c|} 
Blackbe rry \\
RIM \\
\end{tabular}} & \multirow[b]{2}{*}{ LG } & \multirow[b]{2}{*}{ Sams ung } & \multirow[b]{2}{*}{ Otro } \\
\hline & & & & & & & & \\
\hline Inicial & 0.2998066 & 0.2746615 & 0.1470019 & 0.0290135 & 0.0367505 & 0.1121857 & 0.0580271 & 0.0425532 \\
\hline 1 & 0.1547389 & 0.2379110 & 0.0773694 & 0.0502901 & 0.2940039 & 0.0870406 & 0.0599613 & 0.0386847 \\
\hline 2 & 0.1412244 & 0.2129955 & 0.0871548 & 0.0942312 & 0.3164898 & 0.0738178 & 0.0446540 & 0.0294325 \\
\hline 3 & 0.1323614 & 0.2005457 & 0.0902365 & 0.1030663 & 0.3301108 & 0.0719531 & 0.0430333 & 0.0286928 \\
\hline 4 & 0.1300404 & 0.1974865 & 0.0911027 & 0.1063022 & 0.3331278 & 0.0712206 & 0.0423199 & 0.0283999 \\
\hline 5 & 0.1293373 & 0.1965096 & 0.0913599 & 0.1071553 & 0.3341094 & 0.0710266 & 0.0421488 & 0.0283532 \\
\hline 6 & 0.1291412 & 0.1962385 & 0.0914311 & 0.1074105 & 0.3343728 & 0.0709718 & 0.0420940 & 0.0283400 \\
\hline 7 & 0.1290848 & 0.1961600 & 0.0914515 & 0.1074816 & 0.3344494 & 0.0709567 & 0.0420790 & 0.0283370 \\
\hline
\end{tabular}


Con base en la muestra se identifican las razones de cambio: el $43 \%$ de los sujetos atribuyen su cambio a que solo querían cambiar de marca, le había gustado el modelo o bien se lo habían robado o se había dañado el equipo, mientras que otro $40 \%$ respondió que buscaba una mejor tecnología.

Tabla 9. Vectores de participación en equilibrio de las marcas

\begin{tabular}{|r|r|}
\hline Sony Ericsson & $12.91 \%$ \\
\hline Nokia & $19.61 \%$ \\
\hline Motorola & $9.15 \%$ \\
\hline iPhone (Apple) & $10.75 \%$ \\
\hline Blackberry (RIM) & $33.45 \%$ \\
\hline LG & $7.10 \%$ \\
\hline Samsung & $4.21 \%$ \\
\hline Otro & $2.83 \%$ \\
\hline
\end{tabular}

Figura 3. Participaciones de mercado de las marcas

La Figura 3 presenta gráficamente el comportamiento de las participaciones de las marcas en el tiempo.
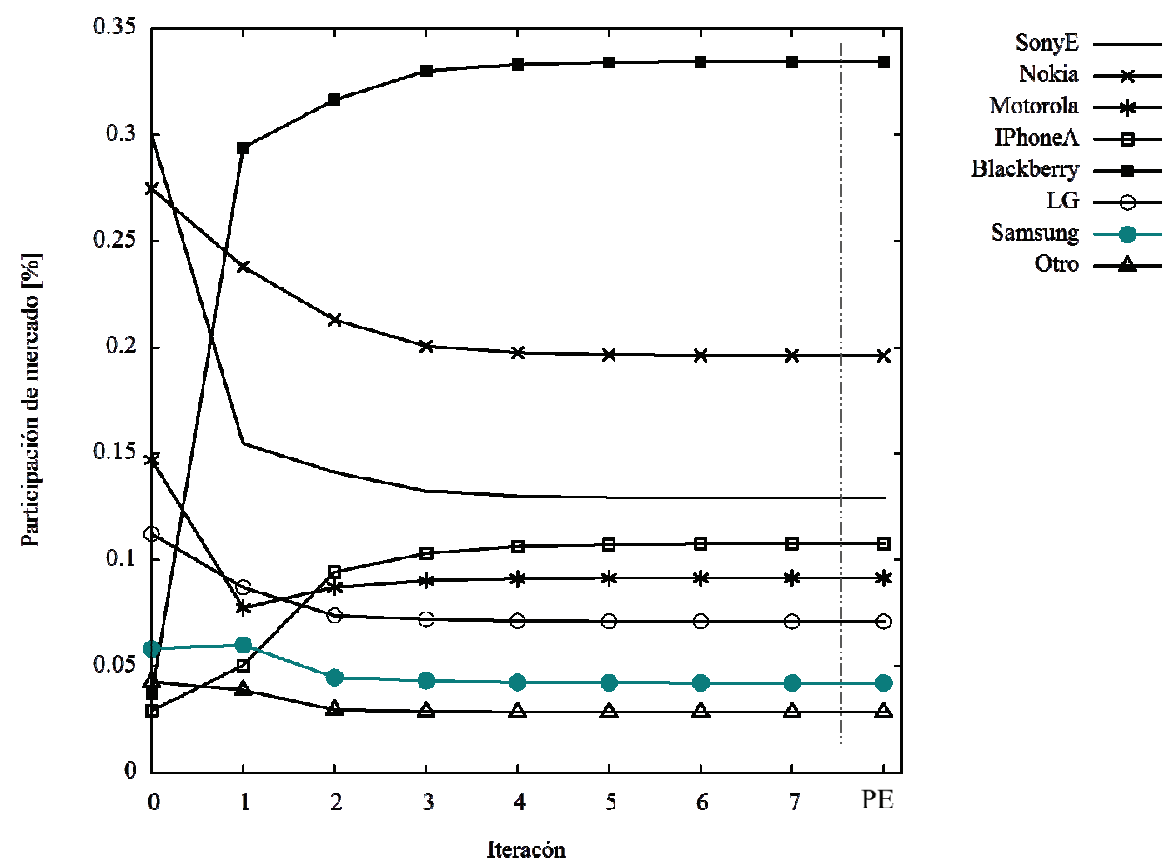
Al comparar los valores de participación de mercado de las primeras iteraciones, tanto para las operadoras como para las marcas (Tablas 6 y 8) con los de equilibrio (Tablas 7 y 9), se estima que se alcanza un error porcentual promedio (Hanke, 2009) menor al 1\% en la cuarta iteración para los operadores y en la sexta iteración para las marcas, lo cual representa 9.2 años $(4 * 2.29)$ y 7.5 años $(6 * 1.25)$ respectivamente.

\section{Análisis de segmentación}

El objetivo de la segmentación de mercado es identificar diferencias y posibles pautas que permiten definir estrategias de mercadotecnia, con el objetivo de alterar convenientemente dichas tendencias. A continuación se presenta un análisis de escenarios tendenciales de las participaciones de mercado, tanto para las operadoras como para las marcas, considerando tres criterios de segmentación. Los criterios de segmentación utilizados para el análisis fueron (Tabla 10): género (mujer y hombre), tipo de contrato de teléfono móvil (pre y post pago) y TV de paga en casa (con contrato y sin contrato).

Tabla 10. Criterios de segmentación y tamaño de los segmentos $(\mathrm{n}=518)$

\begin{tabular}{|c|c|c|c|c|}
\hline Género & Mujer & $53.7 \%$ & Hombre & $46.3 \%$ \\
\hline Contrato & Pre pago & $56.2 \%$ & Post pago & $43.8 \%$ \\
\hline TV paga & No & $27.8 \%$ & Sí & $72.2 \%$ \\
\hline
\end{tabular}

Las Tablas 11 y 12 muestran las participaciones iniciales y las de equilibrio de las operadoras y de las marcas, para cada uno de los segmentos considerados. Los cálculos se realizaron tomando las matrices de transición para cada uno de los segmentos. En los Anexos uno y dos se presentan gráficamente las participaciones de las primeras iteraciones y las participaciones en equilibrio para cada segmento de las operadoras y de las marcas.

Entre las empresas operadoras, Telcel mantiene su posición de operadora líder, manteniendo o incrementando su participación sobre todo en el segmento de pre pago en un $18 \%$. Nextel incrementa su participación en forma notable en el segmento de no con TV de paga. Iusacel incrementa su participación en todos los segmentos y sobre todo en el de post pago y mujeres. Las perspectivas de Movistar y Unefon son negativas, ya que disminuyen su participación en todos los segmentos menos en el de no TV de paga para Unefon.

Respecto a las marcas de teléfonos móviles, Sony Ericsson, Motorola, LG y Samsung disminuyen su participación en prácticamente todos los segmentos. iPhone y Blackberry, marcas de alto dinamismo tecnológico, son las que crecen notablemente su participación en todos los segmentos. En el segmento de mujeres y post pago Blackberry logra incrementos de once y trece veces respectivamente. 
Por su parte, iPhone incrementa su participación sobre todo en los segmentos de hombres, con contrato de pre pago y con TV de paga.

Tabla 11. Participaciones iniciales (Po) por segmento

\begin{tabular}{|c|c|c|c|c|c|c|c|}
\hline Po & Total & Pre pago & Post pago & Mujeres & Hombres & TV paga & No TV paga \\
\hline Telcel & $78.19 \%$ & $76.63 \%$ & $80.18 \%$ & $75.90 \%$ & $80.83 \%$ & $78.07 \%$ & $78.47 \%$ \\
\hline Nextel & $4.25 \%$ & $1.03 \%$ & $8.37 \%$ & $5.04 \%$ & $3.33 \%$ & $5.61 \%$ & $0.69 \%$ \\
\hline Iusacell & $3.47 \%$ & $2.41 \%$ & $4.85 \%$ & $4.32 \%$ & $2.50 \%$ & $4.01 \%$ & $2.08 \%$ \\
\hline Movistar & $10.81 \%$ & $16.15 \%$ & $3.96 \%$ & $11.51 \%$ & $10.00 \%$ & $8.29 \%$ & $17.36 \%$ \\
\hline Unefon & $3.28 \%$ & $3.78 \%$ & $2.64 \%$ & $3.24 \%$ & $3.33 \%$ & $4.01 \%$ & $1.39 \%$ \\
\hline Sony Ericsson & $29.98 \%$ & $31.27 \%$ & $28.32 \%$ & $27.34 \%$ & $33.05 \%$ & $28.69 \%$ & $33.33 \%$ \\
\hline Nokia & $27.47 \%$ & $27.15 \%$ & $27.88 \%$ & $30.94 \%$ & $23.43 \%$ & $27.88 \%$ & $26.39 \%$ \\
\hline Motorola & $14.70 \%$ & $11.68 \%$ & $18.58 \%$ & $18.35 \%$ & $10.46 \%$ & $15.82 \%$ & $11.81 \%$ \\
\hline iPhone (Apple) & $2.90 \%$ & $1.03 \%$ & $5.31 \%$ & $3.60 \%$ & $2.09 \%$ & $3.49 \%$ & $1.39 \%$ \\
\hline Blackberry (RIM) & $3.68 \%$ & $3.09 \%$ & $4.42 \%$ & $2.16 \%$ & $5.44 \%$ & $4.29 \%$ & $2.08 \%$ \\
\hline LG & $11.22 \%$ & $12.71 \%$ & $9.29 \%$ & $8.99 \%$ & $13.81 \%$ & $11.53 \%$ & $10.42 \%$ \\
\hline Samsung & $5.80 \%$ & $6.53 \%$ & $4.87 \%$ & $5.40 \%$ & $6.28 \%$ & $5.63 \%$ & $6.25 \%$ \\
\hline Otro & $4.26 \%$ & $6.53 \%$ & $1.33 \%$ & $3.24 \%$ & $5.44 \%$ & $2.68 \%$ & $8.33 \%$ \\
\hline
\end{tabular}

Tabla 12. Participaciones en equilibrio (PE) por segmento

\begin{tabular}{|c|c|c|c|c|c|c|c|}
\hline PE & Total & Pre pago & Post pago & Mujeres & Hombres & TV paga & No TV paga \\
\hline Telcel & $84.74 \%$ & $90.33 \%$ & $79.00 \%$ & $80.62 \%$ & $89.24 \%$ & $83.89 \%$ & $87.09 \%$ \\
\hline Nextel & $3.79 \%$ & $0.81 \%$ & $7.07 \%$ & $5.35 \%$ & $2.10 \%$ & $4.53 \%$ & $1.54 \%$ \\
\hline Iusacell & $6.10 \%$ & $2.80 \%$ & $9.89 \%$ & $8.36 \%$ & $3.63 \%$ & $7.44 \%$ & $2.60 \%$ \\
\hline Movistar & $4.61 \%$ & $4.85 \%$ & $3.57 \%$ & $5.06 \%$ & $4.11 \%$ & $3.70 \%$ & $7.23 \%$ \\
\hline Unefon & $0.75 \%$ & $1.22 \%$ & $0.48 \%$ & $0.61 \%$ & $0.92 \%$ & $0.44 \%$ & $1.54 \%$ \\
\hline Sony Ericsson & $12.91 \%$ & $21.07 \%$ & $8.56 \%$ & $18.44 \%$ & $9.14 \%$ & $11.17 \%$ & $22.86 \%$ \\
\hline Nokia & $19.61 \%$ & $30.55 \%$ & $5.94 \%$ & $20.04 \%$ & $19.46 \%$ & $13.36 \%$ & $35.79 \%$ \\
\hline Motorola & $9.15 \%$ & $8.30 \%$ & $13.34 \%$ & $15.70 \%$ & $4.89 \%$ & $9.50 \%$ & $6.32 \%$ \\
\hline iPhone (Apple) & $10.75 \%$ & $4.32 \%$ & $15.26 \%$ & $4.74 \%$ & $12.89 \%$ & $13.87 \%$ & $0.74 \%$ \\
\hline Blackberry (RIM) & $33.45 \%$ & $12.05 \%$ & $50.85 \%$ & $28.71 \%$ & $38.00 \%$ & $41.57 \%$ & $9.48 \%$ \\
\hline LG & $7.10 \%$ & $11.10 \%$ & $2.10 \%$ & $4.11 \%$ & $8.40 \%$ & $4.41 \%$ & $13.41 \%$ \\
\hline Samsung & $4.21 \%$ & $9.17 \%$ & $0.91 \%$ & $4.79 \%$ & $2.94 \%$ & $3.39 \%$ & $8.99 \%$ \\
\hline Otro & $2.83 \%$ & $3.43 \%$ & $3.05 \%$ & $3.47 \%$ & $4.28 \%$ & $2.73 \%$ & $2.40 \%$ \\
\hline
\end{tabular}

Resulta interesante notar que en los segmentos de pre y post pago, existe una fuerte diferencia entre la distribución de la muestra comparada con la distribución a nivel nacional. En la muestra, el $56.2 \%$ de los universitarios paga su servicio a través de tarjetas pre pago y el resto $(43.8 \%)$ a través de contratos post pago, 
mientras que a nivel nacional las tarjetas de pre pago son usadas en un $86.6 \%$ y los contratos post pago solamente en $13.4 \%$ (COFETEL, 2010).

\section{DISCUSIÓN Y CONCLUSIONES}

El segmento de universitarios en México no es homogéneo, es decir, existen sub segmentos identificables con comportamientos diferentes, por lo que podría ser adecuado desarrollar estrategias de mercadotecnia multi segmento (Kotler, 2001), enfocándose a cada grupo o sub segmento del mercado universitario, atendiendo a sus características particulares.

Basado en el análisis global y por segmento y a partir de información extraída del cuestionario respecto a razones de permanencia y de cambio de operadoras y de marcas, a continuación se presentan algunas pautas que pudieran definir estrategias de mercadotecnia para modificar las tendencias de las empresas participantes de telefonía móvil, modificando básicamente la matriz de transición de cada sub segmento considerado. A partir de la estructura de dicha matriz, se pueden identificar estrategias orientadas a conservar clientes (diagonal principal $\mathrm{T}_{\mathrm{ij}}$ para $\mathrm{i}=$ j) y estrategias para modificar el comportamiento de cambio $\left(T_{i j}\right.$ para $\left.i \neq j\right)$.

El segmento de estudiantes universitarios en México es en general muy dinámico debido a que busca continuamente nuevos y mejores equipos con nuevas capacidades tecnológicas, por lo que la innovación tanto en los servicios ofrecidos por las operadoras como en los propios teléfonos móviles es un componente esencial en la estrategia competitiva en este sector.

Existe una variedad de detonadores de cambio y de compra de teléfonos móviles y contratación de servicios en el segmento de universitarios, los cuales se basan en la búsqueda de uno o más elementos que los satisfagan. Con base en la muestra utilizada en esta investigación, se identificaron los principales elementos detonadores en la decisión de compra, contratación o cambio:
- Comunicación telefónica eficiente
-Cobertura
-Uso eficaz del sistema de mensajes
-Acceso a Internet
-Acceso a redes sociales
-Entretenimiento
-Estatus
-Novedades tecnológicas
- Costo y tipo de contrato
-Servicio al cliente 
Existe un segmento que busca solamente poder hacer y recibir llamadas y mensajes con equipos de telefonía móvil básicos y a precios accesibles, sin interés en contar con acceso a Internet, redes sociales ni características de entretenimiento (este segmento tiene preferencia por las marcas Nokia y Sony Ericsson). Por otro lado, existe un segmento con interés en el estatus y bienes costosos, dispuestos a pagar precios altos por equipos innovadores con sofisticados atributos de entretenimiento (smartphones de marcas como iPhone y Blackberry). El costo es una característica importante en el sector de universitarios, sin embargo la relación costo beneficio se vuelve fundamental ya que existe la disposición de "invertir" más si se obtiene un mayor beneficio, tanto en el servicio como en el equipo.

Existe interés en el segmento de universitarios en general por las promociones de equipos (tal como ofertas por tiempo limitado, cupones online, etc.), promociones en tarifas (doble tiempo aire, recargas gratuitas, planes de usuarios frecuentes, etc.), ofertas por temporada, promociones de Internet ilimitado, promociones y programas de lealtad de marca, tal como programas y premios de usuario frecuente.

El plan de contratación es un elemento que los universitarios consideran en su selección de empresa operadora y la marca. Dado que más del 50\% prefieren pagar a través de contratos pre pago su servicio de telefonía móvil, es importante que la empresa operadora considere variables como la facilidad para el trámite de contratos, aceptar tanto tarjeta de crédito como de débito y hacer planes más a la medida del perfil universitario como minutos gratis a números frecuentes o internet ilimitado, contratar en otros puntos que no sean las sucursales de servicio al cliente de la compañía como en islas en centros comerciales o stands en universidades. Existe interés en que se amplíen los puntos de venta de las tarjetas de pre pago y recargas de tiempo aire como tiendas de conveniencia, cafeterías de universidades y puestos ambulantes.

La Internet es uno de los canales más importantes de comunicación actualmente, por lo que resulta fundamental utilizar este medio de comunicación. Los universitarios tienden a buscar información de forma activa, con el objetivo de estar actualizados en las nuevas características de las operadoras y de los equipos. La tendencia del sector de universitarios es a "estar conectado", lo cual implica que la comunicación dirigida a este segmento se podría realizar principalmente utilizando redes sociales tal como Facebook (banners y un perfil de la compañía), Twitter, YouTube, LinkedIn y My Space. La actualización y mantenimiento de la página de Internet de las empresas operadoras y las marcas deben considerar la inclusión creativa de información relevante, servicios online, facilidad de uso y rapidez. También se puede utilizar publicidad a través de blogs especializados en tecnología, banners y links, que los re-direccionen a la página de Internet de la empresa. Por otro lado, más del $70 \%$ de universitarios hacen uso de TV de paga, por lo que este canal de comunicación también se vuelve importante en este segmento. 
Comparando la dinámica del mercado de marcas de teléfonos móviles con la del mercado de empresas operadoras, dentro del segmento de universitarios en la ciudad de México, se puede apreciar que respecto a las marcas existe una alta migración de sujetos entre éstas con muy baja lealtad de marca. Sin embargo, considerando a las empresas operadoras, se identifica una alta lealtad al líder del segmento (Telcel), aunado a altas probabilidades de cambio de nuevos usuarios hacia el líder, debido, entre otros factores, a que en la muestra se percibe que ofrece una mayor cobertura, a pesar de tener tarifas altas.

Las estrategias de mercadotecnia para los segmentos con poco dinamismo y alta lealtad serán diferentes a las de alto dinamismo y baja lealtad. La combinación de factores como comunicación telefónica eficiente, cobertura, uso eficaz del sistema de mensajes, acceso a Internet y a redes sociales, entretenimiento, estatus, novedades tecnológicas, costo y tipo de contrato y servicio al cliente sienta la base para identificar objetivos estratégicos, establecer la estrategia para lograrlos y definir las etapas para su ejecución.

Resulta imperativo estar pendiente de los cambios dentro de una industria tan dinámica como la de la telefonía móvil en México, con el objetivo de mantener actualizada las matrices de transición de marcas y operadoras. Eventos de alto impacto como el surgimiento de nuevos competidores, las fusiones de empresas, la capacidad de acceso a canales de comunicación, el aprovechamiento de recursos como la investigación y desarrollo y el ofrecimiento de paquetes de servicios pueden ser factores críticos en el establecimiento de estrategias competitivas. 
Anexo 1. Participaciones de mercado por segmento: operadoras

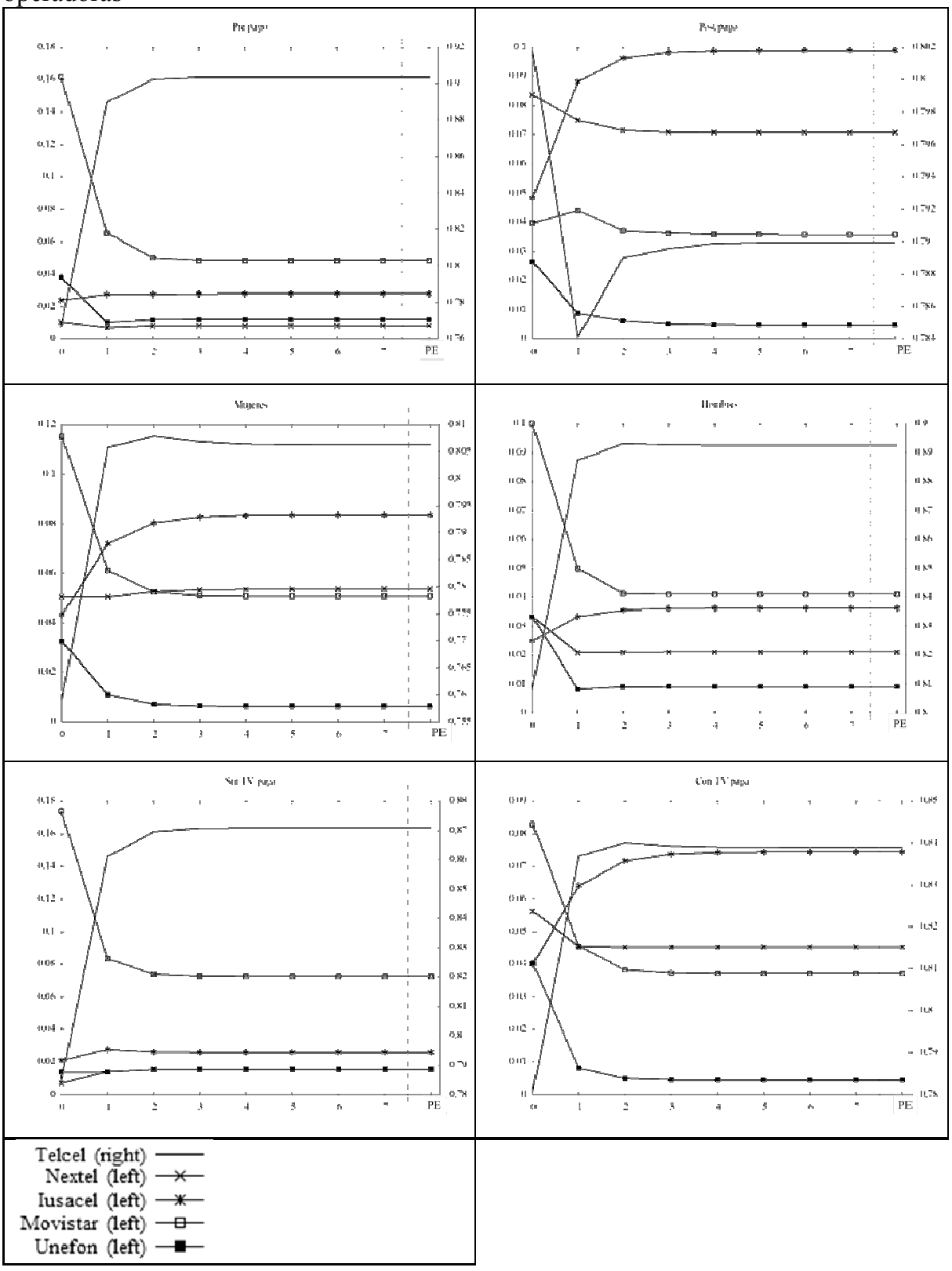


Anexo 2. Participaciones de mercado por segmento: marcas

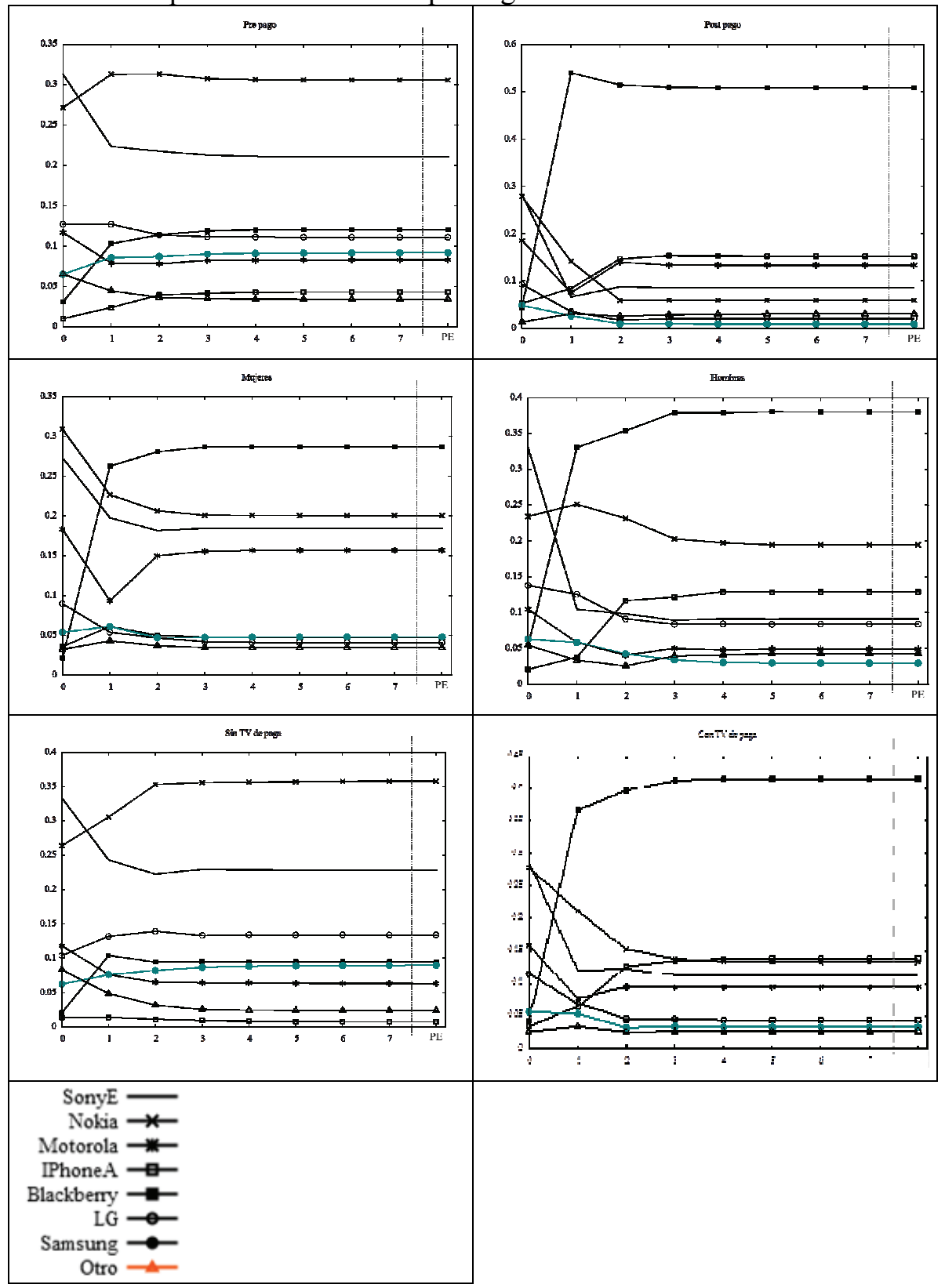




\section{BIBLIOGRAFÍA}

ABUNDIS, F. 2006. Celulares ¿Quién los Usa y Cómo? Datos, Diagnósticos y Tendencias (AMAI). Fecha de consultado: febrero de 2011. Disponible en: www.amai.org

AM. 2012. América Móvil. Consultado: Septiembre 2, 2012. Disponible en: www.americamovil.com/amx/en/cm/about/intro.html?p=28\&s $=34$

AppleWeblog. 2012. Consultado: septiembre de 2012. Disponible en: http://appleweblog.com/2012/05/apple-sobrepasa-a-android-en-estados-unidos

BLATTBERG, R.C. Y SUBRATA K. SEN, S.K. Market Segments and Stochastic Brand Choice Models. Journal of Marketing Research. 13(1), 1976, p. 34-45.

BMI, Business Monitor International. 2011. BMI Mexico Telecommunications Report 2011.

BMI, Business Monitor International. 2010. BMI Global Handset Market: Key Trends \& Opportunities, 2007-2014

CHING, E.S.; FUNG, E.S. Y NG, M.K. A Higher-Order Markov Model for the Newsboy's Problem. The Journal of the Operational Research Society. 54(3), 2003, p. 291- 298.

CHING, W.K.; NG, M.K.; WONG, K.K. Y ALTMAN, E. Customer Lifetime Value: Stochastic Optimization Approach. The Journal of the Operational Research Society. 55(8), 2004, p. 860-868.

COFETEL. 2010. Telefonía Móvil Penetración 1990 - 2010 (MENSUAL). D.F., México.

CIU. The Competitive Intelligence Unit. 2011. La telefonía móvil sigue al alza en México. Noviembre 14 2011. Ariadna Cruz. El Universal. Disponible en: www.eluniversal.com.mx/finanzas/91029.html

DOMENGE, R. y SEVILLA, M. Segmentación de mercados. Nota técnica. Serie de Casos de la Escuela de Negocios del ITAM. México, D.F. 2008.

EFRATI, A. y ANTE, S. Google dobla su apuesta por el sector móvil al comprar Motorola. Wall Street Journal Americas. México, 16 Agosto 2011, p.7.

EHRENBERG, A.S.C. An Appraisal of Markov Brand-Switching Models. Journal of Marketing Research. 2(4), 1965, p. 347-362.

Euromonitor International. Mobile Phones-Mexico.2010a.

Euromonitor International. Smartphones: Not Just iPhones. 2010b.

Euromonitor International. Global Consumer Electronics: Trends, Developments and Insights. 2009. p. 49.

GARTNER. 2010. Gartner Says Worldwide Mobile Phone Sales Grew 35 Percent in Third Quarter 2010; "smartphone" Sales Increased 96 Percent. Consultado: enero de 2011. Disponible en: www.gartner.com/it/page.jsp?id=1466313 
GIVON, M. Y HORSKY, D. Market Share Models As Approximators of Aggregated Heterogeneous Brand Choice Behavior. Management Science. 24(13), 1978, p. 1404-1416.

GONZÁLEZ, E; CASTILLO, A. 2010. PASW como herramienta tecnológica para el análisis de datos cuantitativos. Consultado: agosto de 2011. Disponible en: www.slideshare.net/elgueromisa/pasw-antes-spss-3390102

HANKE, J.E.; WICHERN, D.W. y REITSCH, A.G. Business Forecasting. Ninth Edition. USA, New Jersey: Prentice Hall. 2009.

HARARY, F. Y LIPSTEIN, B. The Dynamics of Brand Loyalty: A Markovian Approach. Operations Research. 10(1), 1961, p. 19-40.

HERNITER, J.D. A Probablistic Market Model of Purchase Timing and Brand Selection. Management Science. Application Series, Part 2, Marketing Management Models. 18(4), 1971, p. 102-113.

HERNITER, J.D. y MAGEE, J.F. Customer Behavior as a Markov Process. Operations Research. 9(1), 1961, p. 105-122.

HOTH, J. El dilema de Nextel. Reforma. México. 17 Agosto 2011, p.4.

INEGI. Instituto Nacional de Estadística y Geografía. Censo de Población y Vivienda 2010. INEGI: México. 2010.

item Software. 2012. Consultado: septiembre de 2012. Disponible en: itemsoft.com/markov.html

KELLEY, A.C. Y WEISS, L.W. Markov Processes and Economic Analysis: The Case of Migration Econometrica. 37(2), 1969, p. 280-297.

KOTLER, P. Dirección de Marketing. México: Pearson Educación. 2001.

LEVIN, R; KIRKPATRICK, C. Quantitative Approaches to Management. 4th Edition International Student Edition. Japan: McGraw-Hill, 1978.

LIPSTEIN, B. A Mathematical Model of Consumer Behavior. Journal of Marketing Research. 2(3), 1965, p. 259-265.

LILIEN, G.L. \& RANGASWAMY, A. Marketing Engineering. Revised Second Edition. USA: Trafford Publishing, 2006.

LÓPEZ, H. 2009. Nivel Socioeconómico AMAI. Consultado: febrero de 2011. Disponible en: www.amai.org

MALHOTRA, N. Investigación de mercados, un enfoque práctico. 5ta Edición. México: Prentice-Hall, 2008.

MARTÍNEZ, G. 2011. Apple está de fiesta, la empresa cumple 35 años. Consultado: abril de 2011. Disponible en: www.excelsior.com.mx/index.php? $m=$ nota\&id_nota $=726564$

MAYORAL, I. 2011. Iusacell y Televisa van contra Telcel. Consultado: abril de 2011. Disponible en: www.cnnexpansion.com/negocios/2011/04/07/iusacell-ytelevisa-van-contra-telcel

Mejía, A. 2011. Empresas de Slim retiran su publicidad de Televisa. Consultado: febrero de 2011. Disponible en: www.eluniversal.com.mx 
MENDOZA YAMASHIRO, K.P. Y HERNÁNDEZ HERRERA, V.L. El mercado de celulares en el segmento de estudiantes universitarios ¿Cómo se comporta este mercado? Tesina. ITAM: México, 2011.

Nextel de México, S.A. de C.V. 2001. Consultado: enero de 2011. Disponible en: www.nextel.com.mx/default.htm

REUTERS. 2011. Samsung, dispuesta a desafiar a Apple. Consultado: enero de 2011. Disponible en: www.cnnexpansion.com/tecnologia/2011/01/18/samsungtecnologicas-rivales-jobs-apple?newsenn $2=\% 5$ btimestamp

REUTERS. 2011. Televisa compra 50\% de Iusacell, paga mil $600 \mathrm{mdd}$. Consultado: abril de 2011. Disponible en: www.excelsior.com.mx/index.php? $m=$ nota\&id_nota $=728006$

STYAN, G.P.H. Y SMITH, H. 1964. Markov Chains Applied to Marketing. Journal of Marketing Research. 1(1), 1964, p. 50-55.

TUIRÁN, R. 2010. La educación superior en México: avances, rezagos y retos. Consultado: marzo de 2011. Disponible en: www.ses.sep.gob.mx

VILCASSIM, N.J. Y JAIN, D.C. Modeling Purchase-Timing and Brand-Switching Behavior Incorporating Explanatory Variables and Unobserved Heterogeneity. Journal of Marketing Research. 28(1), 1991, p. 29-41.

WHITAKER, D. The Derivation of a Measure of Brand Loyalty Using a Markov Brand Switching Model. The Journal of the Operational Research Society. 29(10), 1978, p. 959- 970. 